\title{
User-driven Service Innovation in a Smarter City Living Lab
}

\author{
Sauro Vicini \\ City of the Future Living Lab \\ Fondazione Centro San Raffaele \\ Italy \\ vicini.sauro@hsr.it
}

\author{
Sara Bellini \\ City of the Future Living Lab \\ Fondazione Centro San Raffaele \\ Italy \\ bellini.sara@hsr.it
}

\author{
Alberto Sanna \\ eServices for Life and Health \\ Fondazione Centro San Raffaele \\ Italy \\ sanna.alberto@hsr.it
}

\begin{abstract}
This paper presents the case for San Raffaele Scientific Institute's (FCSR) City of the Future Living Lab in Milan as both a virtual and real research environment and community, through which to explore and discuss how a CoCreation methodology in a Living Lab setting can be applied to the innovation of services and the potential of Internet of Things technologies in this process. The paper is explorative and induced from an ongoing development and practical implementation of the Living Lab in FCSR. The paper presents an overview of the methodology and tools implemented in all the phases of the Co-Creation process and presents the case of Living Labs as user- driven open innovation ecosystems for services for future Smarter Cities.
\end{abstract}

Keywords- Service Innovation, eServices, Living Lab, Smart Cities, Internet of Things, Co-Creation, User-driven Open Innovation

\section{INTRODUCTION}

Innovation is the talk of the town, though ways to hone and foster it are complex and require a methodology that must continuously adapt and evolve. For this reason, the San Raffaele Scientific Institute (FCSR) in Milan has added among its significant assets a specific unit oriented to Information Technology applications in the service domain. This unit, called eServices for Life and Health has set up the City of the Future Living Lab, with the aim of exploring the Co-Creation methodology for the innovation and deployment of tomorrow's eServices. By involving users directly in the Co-Creation process of services within a real city environment, a user-driven open innovation ecosystem was created thus bridging the gap between fundamental and applied research. By integrating the strengths and engaging the efforts of users, stakeholders, researchers, Small and Medium Enterprises (SMEs) and policy makers, the City of the Future Living Lab is proving to be a successful breeding ground for disruptively innovative eServices, the foundations for future Smarter Cities.

\section{Smart Cities, Internet of Things AND Service DESIGN}

In order to understand why the concept of Smart Cities, Internet of Things and Service Design are interconnected, and what role they play and values they bring to a Living $\mathrm{Lab}$ environment, it is useful to identify the features of all three.
Contemporary literature offers an array of terms used to define the concept of Smart City, and an overview of most common definitions follows. The report published by the Centre of Regional Science at Vienna University of Technology in 2007 [1] offers a holistic definition of Smart City, which encompasses a series of very valuable characteristics that City of the Future Living Lab finds equally important. For a city to be considered a Smart City, it must call for the cooperation of a multitude of fields of activities including industry, education, community participation, technical infrastructure, and various 'soft factors': "A Smart City is a city well performing in a forward-looking way in six characteristics (Smart Economy, Smart People, Smart Governance, Smart Mobility, Smart Environment, Smart Living), built on the smart combination of endowments and activities of self-decisive, independent and aware citizens."

If analyzed, the above-mentioned definition can be broken down into the following characteristics. A Smart City must have well-developed connectivity obtained through a networked infrastructure [2] [3]. High-tech and creative industries (known as 'soft infrastructure') [4] must emerge from this fertile foundation and over time attract new businesses and investments therefore producing both urban growth and a positive socio- economic performance [5]. Concurrently, strong efforts must go into investing in social and relational capital, with the aim of creating a community that can successfully embrace technology and learn to comprehend, adapt and innovate it [6]. This in turn sets the base for social inclusion of urban residents within public services. And finally, for a city to be considered Smart, efforts must also go into investing in social and environmental sustainability, therefore successfully guaranteeing the safe and renewable use of natural heritage.

The idea at the heart of Internet of Things, which is very similar to the definition of Smart City, is that all things and all environments can be improved from a functional point of view via the embedding of technology that remains invisible to the eye of the users, which enables both products and environments to become smart [7]: meaning that they can gather data (or enable someone to gather data via them) from their surroundings, producing what Fleisch [8] calls highresolution data (or real-time data, essential in management and improvement of systems), as well as communicating amongst themselves as well as with humans. Internet of Things (IoT) is a strongly evolving field and it is key to keep 
in mind that in the future it will be open, scalable, flexible, secure, and customizable by its users, profoundly usercentric.

Service Design is the activity of arranging and managing intangible goods such as people, infrastructures, communication and products, for the attainment of users' goals and the fulfillment of their needs. The aim of this process, as expressed by the Service Design Network Manifesto [9], is to create services that are useful, useable, desirable, efficient and effective, based on a human-centered and holistic approach that focuses on the user experience whilst integrating team-based interdisciplinary approaches and methods, in ever-learning cycles.

The City of the Future Living Lab presents a unique context in which to execute Information and communications technology (ICT)-based services (or eServices), for it is indeed a melting pot of all of the above features: a seamlessly interconnected ecosystem where products and environments interact among themselves across a number of ICT and IoT-enabled services, with the objective of not only empowering users, but also catalyzing the innovation process and bringing about positive social, economic and environmental change for governments, industries and citizens through user active participation. A Smart City context for a living lab provides access not only to a wide set of users and stakeholders, but also to continuous stimuli since the scenarios available for analysis are variegated and concern all aspects of everyday life. Such a fertile setting enhances the process of service innovation and helps to develop real user-centered services that truly respond to user needs and are able to permeate the real world.

At the same time, City of the Future living lab believes that the Internet of Things can be considered a powerful means through which to improve the value chain between users, products and environments and therefore create better services. Providing adaptable, scalable and personalized services, or eServices, based on meaningful data collected via embedded technologies in products and environments pushes the concept of Smart Cities towards a future where Cities are Smarter. Since the City of the Future Living Lab embodies the main qualities of a city (see next chapter for a more exhaustive description), it is essential to embrace all three elements, and therefore Smart City, Internet of Things and Service Design, so as to create an innovation continuum that is evolutionary and potentially disruptive.

\section{A LiVING LAB FOR SMARTER Cities}

FCSR has recently set up the City of the Future Living Lab (http://www.cityofthefuturelab.org), both a virtual as well as real research environment and community. The Living Lab is managed and organized by "eServices for Life and Health" (http://www.eservices4life.org), a department of FCSR specialized in the application of Information Technology to health, with the aim of developing and delivering services to the hospital's infrastructure as well as fostering innovation across numerous domains and disciplines. The Living Lab follows along the conceptual framework presented by ESoCE-Net in which user-driven and open innovation is fully integrated within the co-creation process of new services, products and societal infrastructures [10]. As of 2012, City of the Future Living Lab has been awarded membership of the Enoll network (European Network of Living Labs: http://www.openlivinglabs.eu).

The City of the Future Living Lab is an ecosystem where a multitude of stakeholders and partners can work alongside each other sharing knowledge whilst interacting with a wide variety of ICTs, therefore creating a fertile ground for innovation and cross-disciplinary research and communication. The City of the Future Living Lab is a miniature version of a city, with a hospital, an university, a hotel, stores and offices, a supermarket, an automatic metro rail and bus service as well as streets and parks. In such a context, over 580,000 patients are hospitalized per year, whilst the hospital provides work to over 960 doctors, 1,500 staff and 240 technicians, whilst the university counts over 3,000 people yearly between students and staff per year. This means that the Living Lab can access 20,000 visitors daily. and can articulate itself in numerous scenarios. The aim of the City fo the Future Living Lab is of understanding, studying and measuring the interaction dynamics among users and services offered and the potential of Internet of Things technologies.

A selection of four scenarios will be covered in this short paper to describe four very different forms of eServices. The latter are a result of a process of fine-tuning with the final users and constitute one of the use cases of ELLIOT Experiential Living Lab for the Internet of Things - an FP7ICT funded project for the observation and evaluation of the user experience in a Living Lab setting. This project is expected to dramatically increase the adoption of Internet of Things and to enhance the potential of collaborative innovation for the discovery of innovative Internet of Things, application/service opportunities in bridging the technological distance with users/citizens.

\section{FCSR'S CO-CREATION METHODOLOGY}

The research methodology implemented for the study of City of the Future scenarios derives from and reinterprets the widely recognized Living Lab process. The Co-Creation process ideated and developed by City of the Future Living $\mathrm{Lab}$ is based on four concurrent phases (cf. Figure 1): CoDesign, Implementation, Experimentation, and Evaluation. Since this approach is an iterative and reflective one, a starting point is not defined because each stage of the CoCreation methodology can be commenced at any stage of the traditional innovation and development process.

Living Labs are innovation environments that focus on user communities embedded within "real life" situations and environments. The fundamental concept at the base of a Living Labs is to gain direct and unfiltered access to users' ideas, experiences, and knowledge, based on their daily needs and desire of feeling supported by products, services, or applications. Users are directly involved in co- designing, implementing, experimenting and evaluating new ideas, concepts and technological artifacts related to Internet of Things, applications and services. The Living Lab CoCreation methodology is an approach that focuses on making stakeholders and users constructive and active participants in 


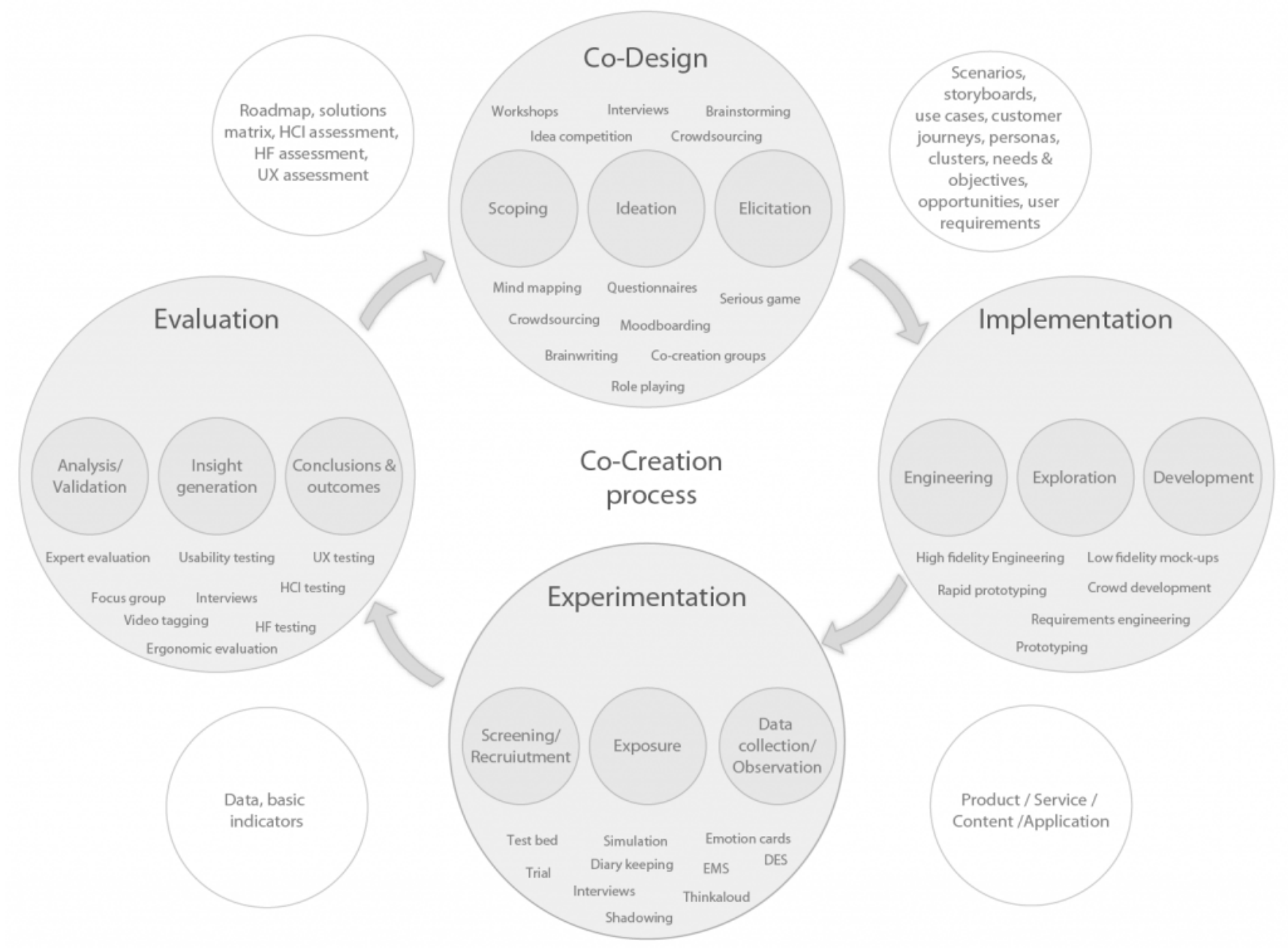

Figure 1. Articulation of the Co-Creation process adopted in FCSR's City of the Future Living Lab

the definition and construction of an artifact, be it a product, an interface or a service, with the aim of improving and building value into them. Users and stakeholders include patients and their families, the general public, and a vast number of experts: technical staff (i.e. City of the Future Living Lab experts which include designers, IT and mechanical engineers, project managers, ethnographers to name a few), medical experts (pediatricians, psychologists, nutritionists, physiologists, etc.), as well as sociologist, dieticians, teachers, marketers, and so on. These individuals are all involved from the early stage of the Living Lab process and throughout its cycle. Insights are gathered directly from the users in order to define and implement realistic, useful, desirable and effective artifacts.

Co-design is a phase that is made up of three sub-phases: scoping, ideation and elicitation. It is based on the user centered design model, which focuses on understanding the end user in order to identify user needs, desires and habits that can guide each stage of the design process so that the solutions developed can truly permeate every-day life. The process starts with an analysis of the selected scenario, in which users' needs, preference and behaviors are examined through methods such as observation, shadowing and interviewing, alongside a careful research into market segmentations and trends. The Co-Design phase also involves activities (such as co-creation groups, brainstorming sessions or idea competitions), where end- users are invited to spontaneously and freely contribute ideas or suggestions on ways to improve or re-invent services, products and interfaces which can be more or less elicited. These ideas, alongside a set of user requirements, form the foundation for the following phases of the Living Lab process,

The next phase, the Implementation phase, refers to the process of developing and making of prototypes and mockups that represent users' suggestions and ideas emerged during the Co-Design phase. There are three main subphases: engineering, exploration and development. Prototypes and mock-ups are developed by the eServices for Life and Health research team and evaluated by specialized experts and stakeholders [cf. Figure 2] as well as end-users. In this manner, a well- balanced set of insights can be gathered and constructively contribute to the fine-tuning of the prototypes before they are distributed in real-life contexts during the Experimentation phase. 
The Experimentation stage consists of having a wide range of users interact and experience the fine-tuned concepts from the previous phases in real and concrete environments and contexts. The reason for this is to verify the effectiveness, utility, appeal and liking of the concepts
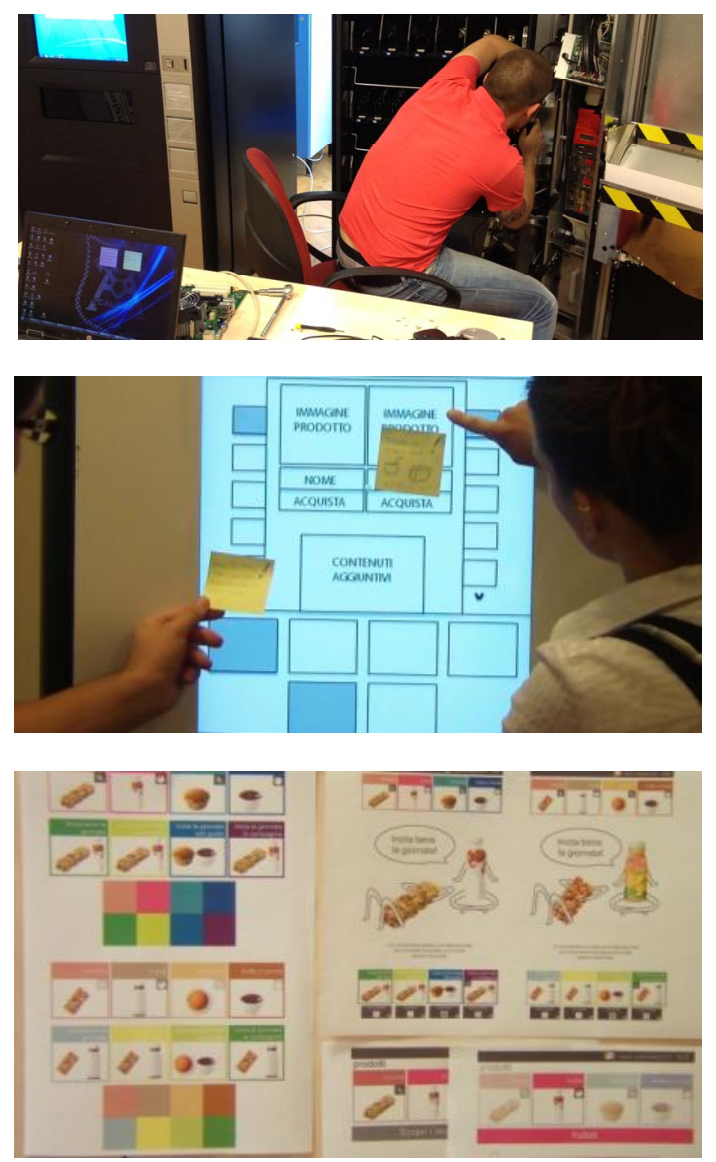

Figure 2. Images illustrating different sorts of activities which can take place in the Implementation phase of the Co-Creation process

that have emerged from the previous two stages with the aim of improving them even more. In this phase, users are recruited usually as volunteers from the City of the Future Living Lab, they are exposed to the concepts developed and data (both qualitative and quantitative) is collected both by research team members as well as through an IoT system (integrated into the concepts or embedded in the surroundings of the Living Lab).

The methodology adopted throughout the Experimentation phase is based on eServices for Life and Health Unit's past experience acquired via Clinical Trials. The knowledge and skills attained via scrupulous and attentive traditional pharmacological and medical research, has allowed the team to design a Testbed-based protocol to be used for less critical research. It consists of a series of ethnographic methods [cf. Figure 3] where users are carefully observed and monitored in either a shielded or realistic environment so as to collect authentic insights for the Evaluation phase.

The Evaluation phase is a result of the analysis of the collected and observed data from the Experimentation phase, and is composed of three sub-phases: analysis, insight generation and conclusions. This phase consists in the analysis of the feedback and data collected, by research members and experts, and the translation of these into insights. This process involves both quantitative data collected via the previously-mentioned IoT system, as well as qualitative data collected through more traditional methods (such as questionnaires or focus groups). These insights are then distilled and compared to the insights collected throughout the entire process and conclusions are drawn.
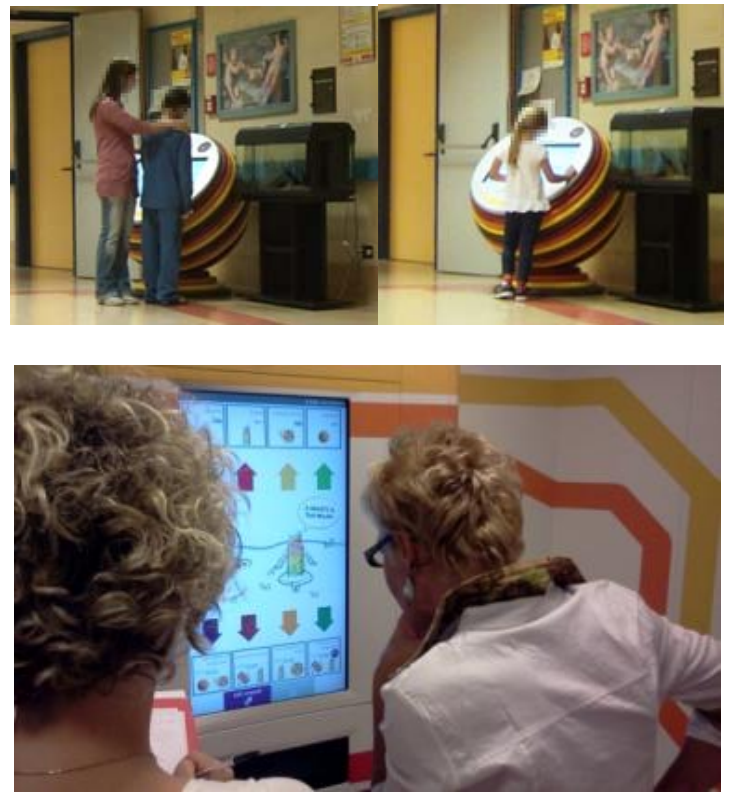

Figure 3. Observation of users during the Experimentation phase

\section{INTERNET OF THINGS ESERVICES TESTED IN CITY OF THE FUTURE LIVING LAB}

The City of the Future Living Lab supports and endorses an extended concept of well-being, and believes that the eServices deployed should promote users' mental, emotional, physical and social well-being as conveyed by the World Health Organization widely recognized statement: "health is a state of complete physical, mental and social well-being and not merely the absence of disease or infirmity". For this reason the eServices developed are centered on the user, his/her well-being, and the experiences they can provide, and are applied to a number of different scenarios which are briefly described in the following sections. 


\section{A. Edutainment Service}

The Edutainment Service [cf. Figure 4] articulates itself in the pediatric department of FCSR. It involves an interactive totem with a touch screen monitor and an easy-touse interface suitable for children, and allows hospitalized children to access educational games so that they can continue learning during their stay in hospital [11]. The more children play with these games, the more they can acquire points to play with purely entertaining games. Each login is age specific and offers games that are designed for the child in question. This approach allows to control the applications the children can use and provide the proper educational and entertainment contents based on each age group. The children can also order their own meals via the Interactive Totem, which not only gives them the chance of feeling empowered and active, it is also a way to convey in a fun way information about healthy eating and the importancing of physical activity.

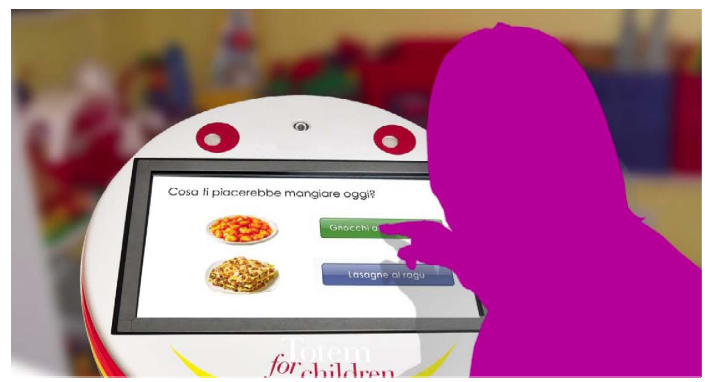

Figure 4. Part of FCSR's Edutainment Service: an Interactive Totem

\section{B. Headings Healthy Lifestyle Choices Service}

The Healthy Lifestyle Choices Service offers a service for the promotion of healthy lifestyles, which stimulates users to reflect upon the benefits related to healthy living through a new concept of vending machines [cf. Figure 5]. Indeed, these vending machines not only provide goods, but also the opportunity for its users to gather and to be entertained - when the machine is not in use, it operates as an entertainment tool displaying musical performances [12]. The vending machines feature an LCD touch screen panel in place of the usual glass front, through which all sorts of users (children through to senior users) can interact with the machine easily and comfortably. Users can view the selected product, its ingredients and nutritional details via the screen.

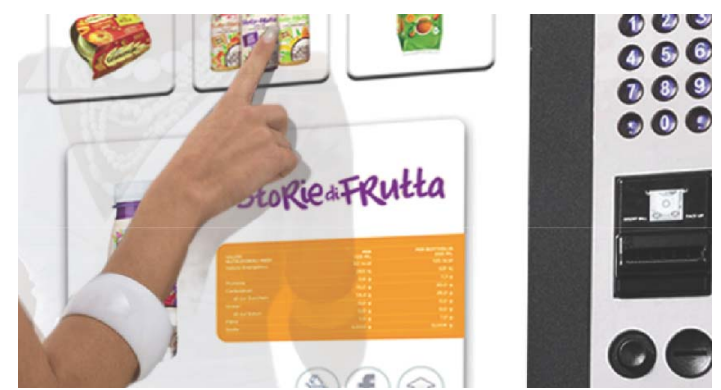

Figure 5. A user interacting with FCSR's Interactive Vending Machine

The vending machines are able to track all transactions and interactions, and record their usage history. Moreover, the vending machines are equipped with a webcam and a microphone that can register video and audio footage, which in turn provide information regarding the social interactions among users standing around them.

\section{Conscientious Tourism Service}

The Conscientious Tourism Service articulates itself around the Vainbici Web Portal (www.vainbici.it) conceived for the promotion of initiatives for healthier and environmentally friendly lifestyles whilst providing innovative services for pedestrians' and cyclists' mobility. This digital platform [cf. Figure 6] was designed to promote the exchange and sharing of information and digital content related to the world of bikes and cycling, including audiovisual multimedia contents. Users can access the website anonymously or as registered users, and can navigate a vast number of services including news related to the world of wheeled vehicles, interesting itineraries for professionals as well as for tourists, maps and altitude profiles of paths, location of tourist services available on the territory or fun initiatives in which to participate.

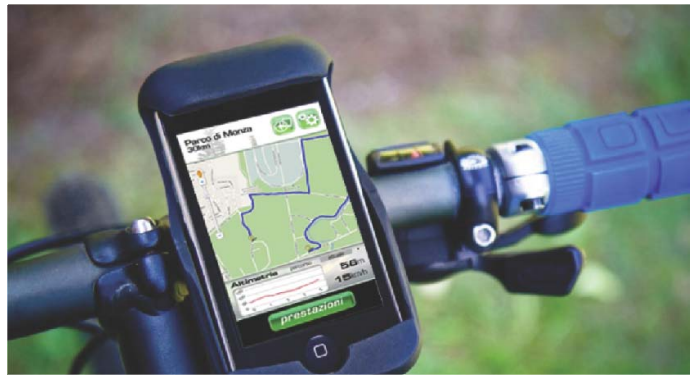

Figure 6. The digital platform of Vainbici via its Smartphone application

\section{InfoMobility Service}

The InfoMobility Service concentrates around users' mobility to and from the FCSR, with particular focus on the scientific institute's automatic shuttle line. The aim is to improve the flow of people by supporting patients, visitors, 
staff and students in their travel to and from the FCSR's grounds and navigation.

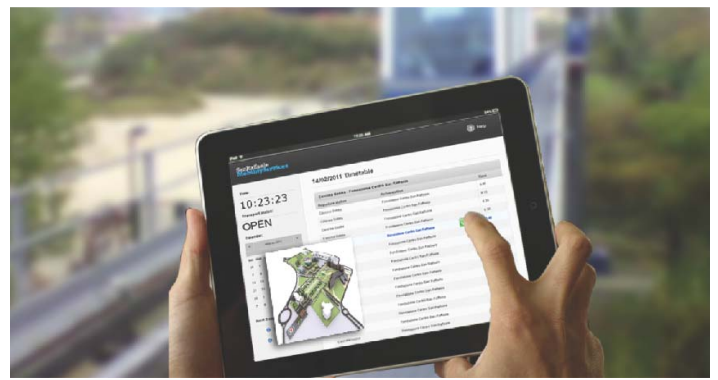

Figure 7. A user accessing the shuttle line's timetable as moment of the InfoMobility Service

Users can access a dedicated website, either from a pc station or from a mobile device [cf. Figure 7], and retrieve dedicated information including the timetable of the shuttle line, news about the condition of the automatic line, and realtime updates on the next connections from and towards the San Raffaele Scientific Institute. They can also program their journey in advance and access information on bus lines, parking spaces and cycling facilities.

\section{CONCLUSIONS}

An innovation process where potential and final users are actively involved throughout makes it possible that the eServices produced are truly user-driven and user-centered. Living Labs are the most natural and effective setting for the co-design, implementation, experimentation and evaluation of eServices, and are proving to be the most pragmatic, functional and economically convenient way to offer future users of services solutions.

FCSR's City of the Future Living Lab is a virtual as well as real research environment and community that embodies a Smart City and with its integrated IoT system is able to involve users throughout the innovation process at a new level. In such a context, a number of eServices are being ideated and offered across different scenarios, with the objective of exploring the Co-Creation method and the effectiveness of an IoT system in developing truly satisfying and usable services able to develop and permeate Smarter Cities.

\section{REFERENCES}

[1] Giffinger, R., et al. (2006) Smart cities - Ranking of European medium-sized cities', Centre of Regional Science, Vienna. WWW page. cities.eu/download/smart_cities_final_report.pdf

[2] Hollands, R. G. (2008) Will the real smart city please stand up? City, Vol. 12, No. 3

[3] Caragliu, A., Del Bo, C., Nijkamp, P. (2009) Smart cities in Europe'. Serie Research Memoranda 0048, VU University Amsterdam, Faculty of Economics, Business Administration and Econometrics

[4] Florida, R. L. (2002) The rise of the creative class: and how it's transforming work, leisure, community and everyday life, Basic Books, New York

[5] Shaffers, H., et al. (2011) Smart Cities and the Future Internet: Towards Cooperation Frameworks for Open Innovation, Lecture Notes in Computer Science, Volume 6656, The Future Internet. WWW page http://www.springerlink.com/content/h6v7x10n5w7hkj23/

[6] Caragliu, A., Del Bo, C., Nijkamp, P. (2009) Smart cities in Europe' Serie Research Memoranda 0048, VU University Amsterdam, Faculty of Economics, Business Administration and Econometrics

[7] Vermesan, O. et al (2009) Internet of Things - Strategic Research Roadmap. WWW page: http://sintef.biz/upload/IKT/9022/CERPIoT\%20SRA_IoT_v11_pdf.pdf

[8] Fleisch, E. (2006) What is the Internet of Things? An Economic Perspective. WWW page. http://www.autoidlabs.org/uploads/media/AUTOIDLABS-WPBIZAPP-53.pdf

[9] Service Design Manifesto. WWW page: http://www.service-designnetwork.org/system/files/media/sdn\%20Manifesto.pdf

[10] Santoro, R., Conte, M. (2010) Living Labs in Open Innovation Functional Regions, ESoCE-NET White Paper. WWW page: www.esoce.net/Living\%20Labs\%20in\%20Functional\%20Regions $\% 2$ $0-\% 20$ White $\% 20$ Paper.pdf

[11] Vicini, S. et al. (2012) "A Living Lab for Internet of Things Vending Machines", ImViReLL conference paper

[12] Vicini. S. et al. (2012), "An internet of things enabled interactive totem for children in a living lab setting", 18th International ICE Conference on Engineering, Technology and Innovation (ICE), Munich 